\title{
Brain Tumor Segmentation and Survival Prediction Using Multimodal MRI Scans With Deep Learning
}

\author{
Li Sun ${ }^{1}$, Songtao Zhang ${ }^{1}$, Hang Chen ${ }^{1}$ and Lin Luo ${ }^{1,2 *}$ \\ ${ }^{1}$ School of Innovation and Entrepreneurship, Southern University of Science and Technology, Shenzhen, China, ${ }^{2}$ College of \\ Engineering, Peking University, Beijing, China
}

Gliomas are the most common primary brain malignancies. Accurate and robust tumor segmentation and prediction of patients' overall survival are important for diagnosis, treatment planning and risk factor identification. Here we present a deep learning-based framework for brain tumor segmentation and survival prediction in glioma, using multimodal MRI scans. For tumor segmentation, we use ensembles of three different 3D CNN architectures for robust performance through a majority rule. This approach can effectively reduce model bias and boost performance. For survival prediction, we extract 4,524 radiomic features from segmented tumor regions, then, a decision tree and cross validation are used to select potent features. Finally, a random forest model

OPEN ACCESS

Edited by: Spyridon Bakas, University of Pennsylvania, United States

Reviewed by:

Adriano Pinto, University of Minho, Portugal Dong-Hoon Lee, University of Sydney, Australia

*Correspondence:

Lin Luo

luol@pku.edu.cn

Specialty section: This article was submitted to Brain Imaging Methods, a section of the journal

Frontiers in Neuroscience

Received: 26 April 2019

Accepted: 22 July 2019

Published: 16 August 2019

Citation:

Sun L, Zhang S, Chen H and Luo L (2019) Brain Tumor Segmentation and

Survival Prediction Using Multimodal MRI Scans With Deep Learning.

Front. Neurosci. 13:810. doi: 10.3389/fnins.2019.00810 is trained to predict the overall survival of patients. The 2018 MICCAI Multimodal Brain Tumor Segmentation Challenge (BraTS), ranks our method at 2nd and 5th place out of $60+$ participating teams for survival prediction tasks and segmentation tasks respectively, achieving a promising $61.0 \%$ accuracy on the classification of short-survivors, mid-survivors and long-survivors.

Keywords: survival prediction, brain tumor segmentation, 3D CNN, multimodal MRI, deep learning

\section{INTRODUCTION}

A brain tumor is a cancerous or noncancerous mass or growth of abnormal cells in the brain. Originating in the glial cells, gliomas are the most common brain tumor (Ferlay et al., 2010). Depending on the pathological evaluation of the tumor, gliomas can be categorized into glioblastoma (GBM/HGG), and lower grade glioma (LGG). Glioblastoma is one of the most aggressive and fatal human brain tumors (Bleeker et al., 2012). Gliomas contain various heterogeneous histological sub-regions, including peritumoral edema, a necrotic core, an enhancing and a non-enhancing tumor core. Magnetic resonance imaging (MRI) is commonly used in radiology to portray the phenotype and intrinsic heterogeneity of gliomas, since multimodal MRI scans, such as T1-weighted, contrast enhanced T1-weighted (T1Gd), T2-weighted, and Fluid Attenuation Inversion Recovery (FLAIR) images, provide complementary profiles for different subregions of gliomas. For example, the enhancing tumor sub-region is described by areas that show hyper-intensity in a T1Gd scan when compared to a T1 scan.

Accurate and robust predictions of overall survival, using automated algorithms, for patients diagnosed with gliomas can provide valuable guidance for diagnosis, treatment planning, and outcome prediction (Liu et al., 2018). However, it is difficult to select reliable and potent prognostic features. Medical imaging (e.g., MRI, CT) can provide radiographic phenotype of tumor, and it has been exploited to extract and analyze quantitative imaging features (Gillies et al., 2016). Clinical 
data, including patient age and resection status, can also provide important information about patients' outcome.

Segmentation of gliomas in pre-operative MRI scans, conventionally done by expert board-certified neuroradiologists, can provide quantitative morphological characterization and measurement of glioma sub-regions. It is also a pre-requisite for survival prediction since most potent features are derived from the tumor region. This quantitative analysis has great potential for diagnosis and research, as it can be used for grade assessment of gliomas and planning of treatment strategies. But this task is challenging due to the high variance in appearance and shape, ambiguous boundaries and imaging artifacts, while automatic segmentation has the advantage of fast speed, consistency in accuracy and immunity to fatigue (Sharma and Aggarwal, 2010). Until now, the automatic segmentation of brain tumors in multimodal MRI scans is still one of the most difficult tasks in medical image analysis. In recent years, deep convolutional neural networks (CNNs) have achieved great success in the field of computer vision. Inspired by the biological structure of visual cortex (Fukushima, 1980), CNNs are artificial neural networks with multiple hidden convolutional layers between the input and output layers. They have non-linear properties and are capable of extracting higher level representative features (Gu et al., 2018). Deep learning methods with $\mathrm{CNN}$ have shown excellent results on a wide variety of other medical imaging tasks, including diabetic retinopathy detection (Gulshan et al., 2016), skin cancer classification (Esteva et al., 2017), and brain tumor segmentation (Çiçek et al., 2016; Isensee et al., 2017; Wang et al., 2017; Sun et al., 2018).

In this paper, we present a novel deep learning-based framework for segmentation of a brain tumor and its subregions from multimodal MRI scans, and survival prediction based on radiomic features extracted from segmented tumor sub-regions as well as clinical features. The proposed framework for brain tumor segmentation and survival prediction using multimodal MRI scans consists of the following steps, as illustrated in Figure 1. First, tumor subregions are segmented using an ensemble model comprising three different convolutional neural network architectures for robust performance through voting (majority rule). Then radiomic features are extracted from tumor sub-regions and total tumor volume. Next, decision tree regression model with gradient boosting is used to fit the training data and rank the importance of features based on variance reduction. Cross validation is used to select the optimal number of top-ranking features to use. Finally, a random forest regression model is used to fit the training data and predict the overall survival of patients.

\section{MATERIALS AND METHODS}

\subsection{Dataset}

We utilized the BraTS 2018 dataset (Menze et al., 2015; Bakas et al., 2017a,b,c, 2018) to evaluate the performance of our methods. The training set contained images from 285 patients, including $210 \mathrm{HGG}$ and 75 LGG. The validation set contained MRI scans from 66 patients with brain tumors of an unknown grade. It was a predefined set constructed by
BraTS challenge organizers. The test set contained images from 191 patients with a brain tumor, in which 77 patients had a resection state of Gross Total Resection (GTR) and were evaluated for survival prediction. Each patient was scanned with four sequences: T1, T1Gd, T2, and FLAIR. All the images were skull-striped and re-sampled to an isotropic $1 \mathrm{~mm}^{3}$ resolution, and the four sequences of the same patient had been co-registered. The ground truth of segmentation mask was obtained by manual segmentation results given by experts. The evaluation of the model performance on the validation and testing set is performed on CBICA's Image Processing Portal ipp.cbica.upenn.edu. Segmentation annotations comprise of the following tumor subtypes: Necrotic/non-enhancing tumor (NCR), peritumoral edema (ED), and Gd-enhancing tumor (ET). Resection status and patient age are also provided. The overall survival (OS) data, defined in days, is also included in the training set. The distribution of patients' age is shown in Figure 2.

\subsection{Data Preprocessing}

Since the intensity value of MRI is dependent on the imaging protocol and scanner used, we applied intensity normalization to reduce the bias in imaging. More specifically, the intensity value of each MRI is subtracted by the mean and divided by the standard deviation of the brain region. In order to reduce overfitting, we applied random flipping and random gaussian noise to augment the training set.

\subsection{Network Architecture}

In order to perform accurate and robust brain tumor segmentation, we use an ensemble model comprising of three different convolutional neural network architectures. A variety of models have been proposed for tumor segmentation. Generally, they differ in model depth, filter number, connection way and others. Different model architectures can lead to different model performance and behavior. By training different kinds of models separately and by merging the results, the model variance can be decreased, and the overall performance can be improved (Polikar, 2006; Kamnitsas et al., 2017). We used three different CNN models and fused the result by voting (majority rule). The detailed description of each model will be discussed in the following sections.

\subsubsection{CA-CNN}

The first network we employed was Cascaded Anisotropic Convolutional Neural Network (CA-CNN) proposed by Wang et al. (2017). The cascade is used to convert multi-class segmentation problem into a sequence of three hierarchical binary segmentation problems. The network is illustrated in Figure 3.

This architecture also employs anisotropic and dilated convolution filters, which are combined with multi-view fusions to reduce false positives. It also employs residual connections ( $\mathrm{He}$ et al., 2016), batch normalization (Ioffe and Szegedy, 2015) and multi-scale prediction to boost the performance of segmentation. For implementation, we trained the CA-CNN model using Adam optimizer (Kingma and Ba, 2014) and set Dice coefficient (Milletari et al., 2016) as the loss function. We set the initial 


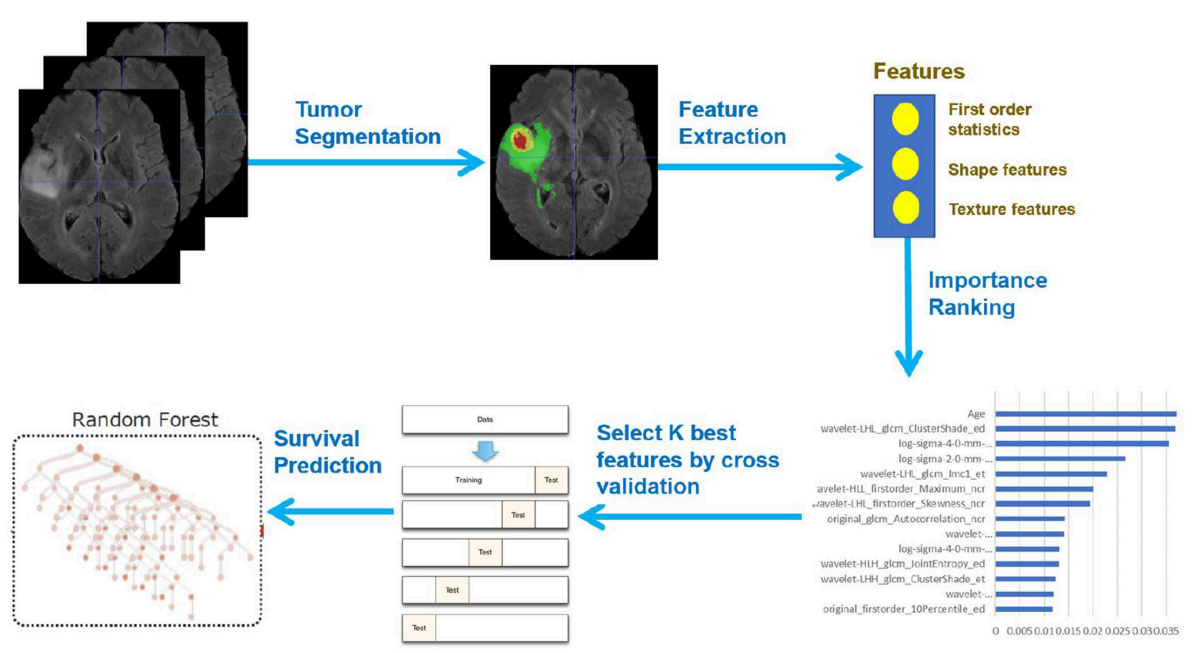

FIGURE 1 | Framework overview.

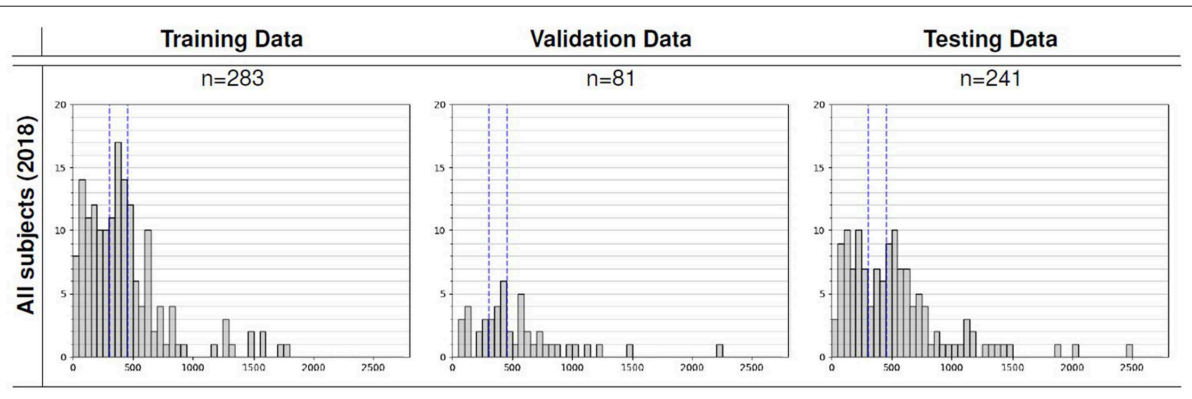

FIGURE 2 | Overall survival distribution of patients across the training, validation, and testing sets.

learning rate to $1 \times 10^{-3}$, weight decay $1 \times 10^{-7}$, batch size 5 , and maximal iteration $30 \mathrm{k}$.

\subsubsection{DFKZ Net}

The second network we employed was DFKZ Net, which was proposed by Isensee et al. (2017) from the German Cancer Research Center (DFKZ). Inspired by U-Net, DFKZ Net employs a context encoding pathway that extracts increasingly abstract representations of the input, and a decoding pathway used to recombine these representations with shallower features to precisely segment the structure of interest. The context encoding pathway consists of three content modules, each has two $3 \times 3 \times 3$ convolutional layers and a dropout layer with residual connection. The decoding pathway consists of three localization modules, each containing $3 \times 3 \times 3$ convolutional layers followed by a $1 \times 1 \times 1$ convolutional layer. For the decoding pathway, the output of layers of different depths are integrated by elementwise summation, thus the supervision can be injected deep in the network. The network is illustrated in Figure 4.

For implementation, we trained the network using the Adam optimizer. To address the problem of class imbalance, we utilized the multi-class Dice loss function (Isensee et al., 2017):

$$
L=-\frac{2}{|K|} \sum_{k \in K} \frac{\sum_{i} u_{i(k)} v_{i(k)}}{\sum_{i} u_{i(k)}+\sum_{i} v_{i(k)}}
$$

where $u$ denotes output possibility, $v$ denotes one-hot encoding of ground truth, $k$ denotes the class, $K$ denotes the total number of classes and $i(k)$ denotes the number of voxels for class $k$ in patch. We set initial learning rate $5 \times 10^{-4}$ and used instance normalization (Ulyanov et al., 2016a). We trained the model for 90 epochs.

\subsubsection{D U-Net}

U-Net (Ronneberger et al., 2015; Çiçek et al., 2016) is a classical network for biomedical image segmentation. It consists of a contracting path to capture context and a symmetric expanding path that enables precise localization with extension. Each pathway has three convolutional layers with dropout and pooling. The contracting pathway and expanding pathway are linked by skip-connections. Each layer contains $3 \times 3 \times$ 3 convolutional kernels. The first convolutional layer has 32 filters, while deeper layers contains twice filters than previous shallower layer. 


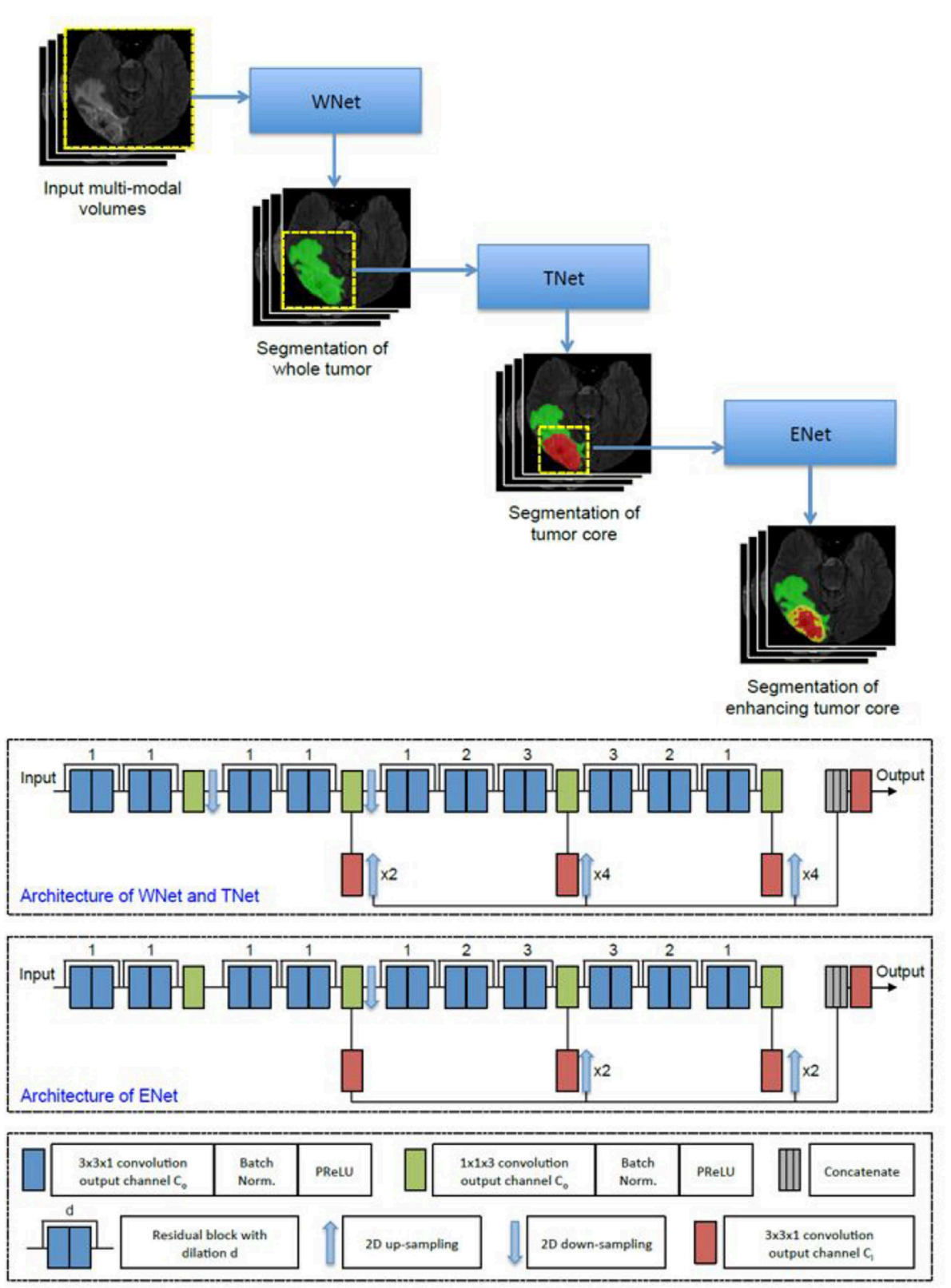

FIGURE 3 | Cascaded framework and architecture of CA-CNN.

For implementation, we used Adam optimizer (Kingma and $\mathrm{Ba}, 2015$ ), and instance normalization (Ulyanov et al., 2016b). In addition, we utilized cross entropy as the loss function. The initial learning rate was 0.001 , and the model is trained for 4 epochs.

\subsubsection{Ensemble of Models}

In order to enhance segmentation performance and to reduce model variance, we used the voting strategy (majority rule) to build an ensemble model without using a weighted scheme. During the training process, different models were trained independently. The selection of the number of iterations in the training process was based on the model's performance in the validation set. In the testing stage, each model independently predicts the class for each voxel, the final class is determined by the majority rule.

\subsection{Feature Extraction}

Quantitative phenotypic features from MRI scans can reveal the characteristics of brain tumors. Based on the segmentation result, we extract radiomics features from edema, non-enhancing solid core and necrotic/cystic core and the whole tumor region respectively using Pyradiomics toolbox (Van Griethuysen et al., 2017). Illustration of feature extraction is shown in Figure 5. 


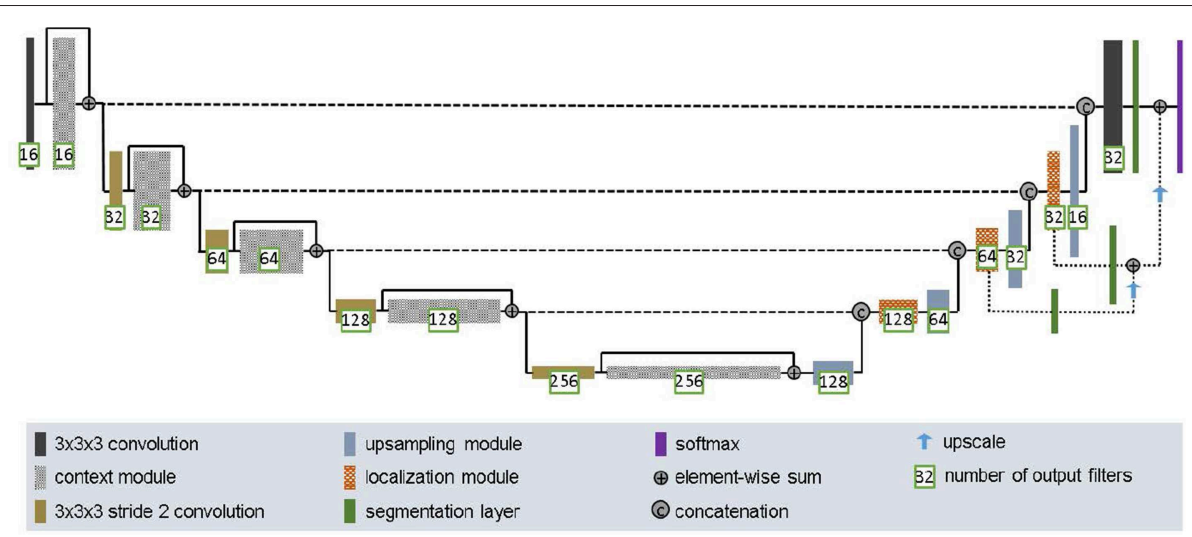

FIGURE 4 | Architecture of DFKZ Net.

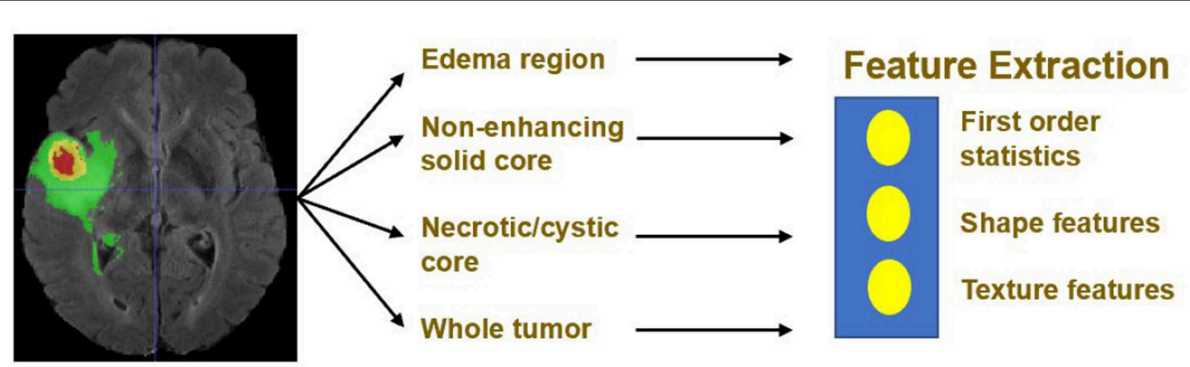

FIGURE 5 | IIlustration of feature extraction.

The modality used for feature extraction is dependent on the intrinsic properties of the tumor subregion. For example, edema features are extracted from FLAIR modality, since it is typically depicted by hyper-intense signal in FLAIR. Non-enhancing solid core features are extracted from T1Gd modality, since the appearance of the necrotic (NCR) and the non-enhancing (NET) tumor core is typically hypo-intense in T1Gd when compared to T1. Necrotic/cystic core tumor features are extracted from T1Gd modality, since it is described by areas that show hyper-intensity in T1Gd when compared to T1.

The features we extracted can be grouped into three categories. The first category is the first order statistics, which includes maximum intensity, minimum intensity, mean, median, 10th percentile, 90th percentile, standard deviation, variance of intensity value, energy, entropy, and others. These features characterize the gray level intensity of the tumor region.

The second category is shape features, which include volume, surface area, surface area to volume ratio, maximum 3D diameter, maximum 2D diameter for axial, coronal and sagittal plane respectively, major axis length, minor axis length and least axis length, sphericity, elongation, and other features. These features characterize the shape of the tumor region.

The third category is texture features, which include 22 gray level co-occurrence matrix (GLCM) features, 16 gray level run length matrix (GLRLM) features, 16 Gray level size zone matrix (GLSZM) features, five neighboring gray tone difference matrix (NGTDM) features and 14 gray level dependence matrix
(GLDM) Features. These features characterize the texture of the tumor region.

Not only do we extract features from original images, but we also extract features from Laplacian of Gaussian (LoG) filtered images and images generated by wavelet decomposition. Because LoG filtering can enhance the edge of images, possibly enhance the boundary of the tumor, and wavelet decomposition can separate images into multiple levels of detail components (finer or coarser). More specifically, from each region, 1131 features are extracted, including 99 features extracted from the original image, and 344 features extracted from Laplacian of Gaussian filtered images, since we used four filters with sigma values 2.0, 3.0, 4.0, 5.0, respectively, and 688 features extracted from eight wavelet decomposed images (all possible combinations of applying either a High or a Low pass filter in each of the three dimensions). In total, for each patient, we extracted $1131 \times 4=4524$ radiomic features, these features are combined with clinical data (age and resection state) for survival prediction. The values of these features except for resection state are normalized by subtracting the mean and scaling it to unit variance.

\subsection{Feature Selection}

A portion of the features we extracted were redundant or irrelevant to survival prediction. In order to enhance performance and reduce overfitting, we applied feature selection to select a subset of features that have the most predictive power. 
Feature selection is divided into two steps: importance ranking and cross validation. We ranked the importance of features by fitting a decision tree regressor with gradient boosting using training data, then the importance of features can be determined by how effectively the feature can reduce intra-node standard deviation in leaf nodes. The second step is to select the optimal

TABLE 1 | Selected most predicative features (WT, edema; TC, tumor core; ET, enhancing tumor; FULL, full tumor volume comprised of edema, tumor core, and enhancing tumor; N/A, not applicable).

\begin{tabular}{|c|c|c|c|}
\hline Extracted from & Name & Subregion & Score \\
\hline wavelet-LHL & glcm_ClusterShade & WT & 0.036912293 \\
\hline log-sigma-2.0mm-3D & gldm_LargeDependenceHighGrayLevelEmphasis & $\mathrm{TC}$ & 0.026591038 \\
\hline wavelet-LHL & glcm_Informational Measure of Correlation & ET & 0.022911978 \\
\hline original image & glcm_Autocorrelation & ET & 0.014204463 \\
\hline wavelet-HHH & gldm_LargeDependenceLowGrayLevelEmphasis & FULL & 0.014085406 \\
\hline log-sigma-4.0mm-3D & firstorder_Mwtian & WT & 0.013031814 \\
\hline wavelet-HLH & glcm_JointEntropy & WT & 0.013023534 \\
\hline wavelet-LHH & glcm_ClusterShade & TC & 0.012335471 \\
\hline
\end{tabular}

TABLE 2 | Evaluation result of ensemble model and individual models.

\begin{tabular}{|c|c|c|c|c|}
\hline Stage & Metric & Enhancing tumor & Whole tumor & Tumor core \\
\hline \multirow{4}{*}{ CA-CNN } & Mean Dice & 0.77682 & 0.90282 & 0.85392 \\
\hline & Mean Hausdorff95(mm) & 3.3303 & 5.41478 & 6.56793 \\
\hline & Sensitivity & 0.81258 & 0.93045 & 0.85305 \\
\hline & Specificity & 0.99807 & 0.99336 & 0.99786 \\
\hline \multirow{4}{*}{ DFKZ Net } & Mean Dice & 0.76759 & 0.89306 & 0.82459 \\
\hline & Mean Hausdorff95(mm) & 5.90781 & 5.60224 & 6.91403 \\
\hline & Sensitivity & 0.80419 & 0.89128 & 0.81196 \\
\hline & Specificity & 0.99833 & 0.99588 & 0.99849 \\
\hline \multirow{4}{*}{ 3D U-Net } & Mean Dice & 0.78088 & 0.88762 & 0.82567 \\
\hline & Mean Hausdorff95(mm) & 7.73567 & 12.63285 & 13.33634 \\
\hline & Sensitivity & 0.84281 & 0.90188 & 0.81913 \\
\hline & Specificity & 0.99743 & 0.99416 & 0.9981 \\
\hline \multirow{4}{*}{ Ensemble model } & Mean Dice & 0.80522 & 0.90944 & 0.84943 \\
\hline & Mean Hausdorff95(mm) & 2.77719 & 6.32753 & 6.37318 \\
\hline & Sensitivity & 0.83064 & 0.90688 & 0.83156 \\
\hline & Specificity & 0.99815 & 0.99549 & 0.99863 \\
\hline
\end{tabular}

The bold values indicate the best performance.

TABLE 3 | Evaluation result of ensemble model for segmentation.

\begin{tabular}{|c|c|c|c|c|}
\hline Stage & Metric & Enhancing tumor & Whole tumor & Tumor core \\
\hline \multirow{2}{*}{ Validation } & Mean Dice & 0.8052 & 0.9044 & 0.8494 \\
\hline & Mean Hausdorff95(mm) & 2.7772 & 6.3275 & 6.3732 \\
\hline \multirow{2}{*}{ Testing } & Mean Dice & 0.7171 & 0.8762 & 0.7977 \\
\hline & Mean Hausdorff95(mm) & 4.9782 & 7.2009 & 6.4735 \\
\hline
\end{tabular}


number of best features for prediction by cross validation. In the end, we selected 14 features and their importance are listed in Table 1. The detailed feature definition can be found at (https://pyradiomics.readthedocs.io/en/latest/features.html), last accessed on 30 June 2018.

Unsurprisingly, age had the most predictive power among all of the features. The rest of the features selected came from both original images and derived images. We also found that most features selected came from images generated by wavelet decomposition.

\subsection{Survival Prediction}

Based on the 14 features selected, we trained a random forest regression model (Ho, 1995) for final survival prediction. The random forest regressor is a meta regressor of 100 base decision tree regressors. Each base regressor is trained on a bootstrapped sub-dataset into order to introduce randomness and diversity. Finally, the prediction from base regressors are averaged to improve prediction accuracy, robustness and suppress overfitting. Mean squared error is used as loss function when constructing individual regression model.
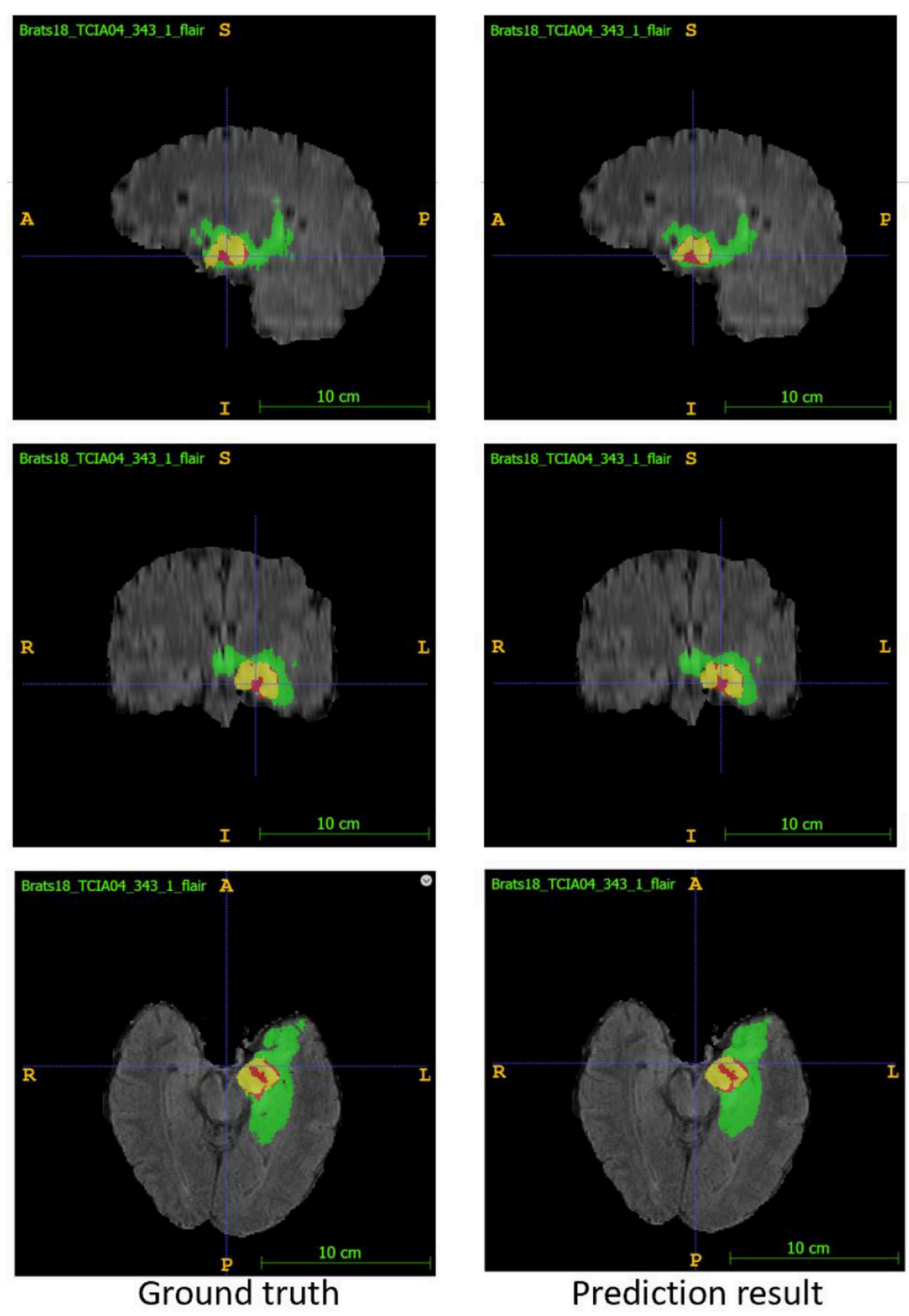

Prediction result

FIGURE 6 | Examples of segmentation result compared with ground truth. Image ID: TCIA04_343_1, Green:edema, Yellow:non-enhancing solid core, Red:enhancing core. 
TABLE 4 | Evaluation result of survival prediction.

\begin{tabular}{lcc}
\hline Stage & Classification accuracy & Median error \\
\hline Validation & $46.4 \%$ & 217.92 \\
Test & $61.0 \%$ & 181.37 \\
\hline
\end{tabular}

\section{RESULTS}

\subsection{Result of Tumor Segmentation}

We trained the model using the 2018 MICCAI BraTS training set using the methods described above. We then applied the trained model for prediction on the validation and test set. We compared the segmentation result of the ensemble model with the individual model on the validation set. The evaluation result of our approach is shown in Table 2. For other teams' performance, please see the BraTS summarizing paper (Bakas et al., 2018). The result demonstrates that the ensemble model performs better than individual models in enhancing tumor and whole tumor, while CA-CNN performs marginally better on the tumor core.

The predicted segmentation labels are uploaded to the CBICA's Image Processing Portal (IPP) for evaluation. BraTS Challenge uses two schemes for evaluation: Dice score and the Hausdorff distance (95th percentile). Dice score is a widely used overlap measure for pairwise comparison of segmentation mask $S$ and $G$. It can be expressed in terms of set operations:

$$
\text { Dice }=\frac{2|S \cap G|}{|S|+|G|}
$$

Hausdorff distance is the maximum distance of a set to the nearest point in the other set, defined as:

$$
d_{\mathrm{H}}(X, Y)=\max \left\{\sup _{x \in X} \inf _{y \in Y} d(x, y), \sup _{y \in Y} \inf _{x \in X} d(x, y)\right\}
$$

where sup represents the supremum and inf the infimum. In order to have more robust results and to avoid issues with noisy segmentation, the evaluation scheme uses the 95 th percentile.

In the test phase, our result ranked 5th out of $60+$ teams. The evaluation result of the segmentation on the validation and test set are listed in Table 3. Examples of the segmentation result compared with ground truth are shown in Figure 6.

\subsection{Result of Survival Prediction}

Based on the segmentation result of brain tumor subregions, we extracted features from brain tumor sub-regions segmented from MRI scans and trained the survival prediction model as described above. We then used the model to predict patient's overall survival on the validation and test set. The predicted overall survival was uploaded to the IPP for evaluation. We used two schemes for evaluation: classification of subjects as long-survivors (>15 months), short-survivors ( $<10$ months), and mid-survivors (between 10 and 15 months) and median error (in days). In the test phase, we ranked second out of $60+$ teams. The evaluation results of our method are listed in Table 4. For other teams' performance, please see the BraTS summarizing paper (Bakas et al., 2018).

\section{DISCUSSION}

In this paper, we present an automatic framework for the prediction of survival in glioma using multimodal MRI scans and clinical features. First, a deep convolutional neural network is used to segment a tumor region from MRI scans, then radiomics features are extracted and combined with clinical features to predict overall survival. For tumor segmentation, we used ensembles of three different 3D CNN architectures for robust performance through voting (majority rule). The evaluation results show that the ensemble model performs better than individual models, which indicates that the ensemble approach can effectively reduce model bias and boost performance. Although the Dice score for segmentation is promising, we noticed that the specificity of the model is much higher than the sensitivity, indicating an under-segmentation of the model. For survival prediction, we extracted shape features, first order statistics, and texture features from segmented tumor sub-region, then used a decision tree and cross validation to select features. Finally, a random forest model was trained to predict the overall survival of patients. The accuracy for three-class classification is $61.0 \%$, which still leaves room for improvement. Part of the reason is that we only had a very limited number of samples (285 patients) to train the regression model. In addition, imaging and limited clinical features may only explain patients' survival outcome partially, too. In the future, we will explore different network architectures and training strategies to further improve our result. We will also design new features and optimize our feature selection methods for survival prediction.

\section{DATA AVAILABILITY}

The datasets analyzed for this study can be found in the BraTS 2018 dataset https://www.med.upenn.edu/sbia/ brats2018/data.html.

\section{AUTHOR CONTRIBUTIONS}

LS and SZ performed the analysis and prepared the manuscript. $\mathrm{HC}$ helped with the analysis. LL conceived the project, supervised and funded the study, and prepared the manuscript.

\section{FUNDING}

Financial support from the Shenzhen Science and Technology Innovation (SZSTI) Commission (JCYJ20180507181527806 and JCYJ20170817105131701) is gratefully acknowledged. 


\section{REFERENCES}

Bakas, S., Akbari, H., Sotiras, A., Bilello, M., Rozycki, M., Kirby, J., et al. (2017a). Segmentation labels and radiomic features for the preoperative scans of the tcga-gbm collection. Cancer Imaging Arch. 286. doi: 10.7937/K9/TCIA.2017.KLXWJJ1Q

Bakas, S., Akbari, H., Sotiras, A., Bilello, M., Rozycki, M., Kirby, J., et al. (2017b). Segmentation labels and radiomic features for the preoperative scans of the tcga-lgg collection. Cancer Imaging Arch. 286. doi: 10.7937/K9/TCIA.2017.GJQ7R0EF

Bakas, S., Akbari, H., Sotiras, A., Bilello, M., Rozycki, M., Kirby, J. S., et al. (2017c). Advancing the cancer genome atlas glioma mri collections with expert segmentation labels and radiomic features. Sci. Data 4:170117. doi: 10.1038/sdata.2017.117

Bakas, S., Reyes, M., et Int, and Menze, B. (2018). Identifying the best machine learning algorithms for brain tumor segmentation, progression assessment, and overall survival prediction in the BRATS challenge. arXiv arXiv:1811.02629.

Bleeker, F. E., Molenaar, R. J., and Leenstra, S. (2012). Recent advances in the molecular understanding of glioblastoma. J. Neuro Oncol. 108, 11-27. doi: 10.1007/s11060-011-0793-0

Çiçek, Ö., Abdulkadir, A., Lienkamp, S. S., Brox, T., and Ronneberger, O. (2016). "3d u-net: learning dense volumetric segmentation from sparse annotation," in Medical Image Computing and Computer-Assisted Intervention - MICCAI 2016, eds S. Ourselin, L. Joskowicz, M. R. Sabuncu, G. Unal, and W. Wells (Cham: Springer International Publishing), 424-432.

Esteva, A., Kuprel, B., Novoa, R. A., Ko, J., Swetter, S. M., Blau, H. M., and Thrun, S. (2017). Dermatologist-level classification of skin cancer with deep neural networks. Nature 542:115-118. doi: 10.1038/nature21056

Ferlay, J., Shin, H.-R., Bray, F., Forman, D., Mathers, C., and Parkin, D. M. (2010). Estimates of worldwide burden of cancer in 2008: Globocan 2008. Int. J. Cancer 127, 2893-2917. doi: $10.1002 /$ ijc. 25516

Fukushima, K. (1980). Neocognitron: a self-organizing neural network model for a mechanism of pattern recognition unaffected by shift in position. Biol. Cybernet. 36, 193-202.

Gillies, R. J., Kinahan, P. E., and Hricak, H. (2016). Radiomics: images are more than pictures, they are data. Radiology 278, 563-577. doi: 10.1148/radiol.2015151169

Gu, J., Wang, Z., Kuen, J., Ma, L., Shahroudy, A., Shuai, B., et al. (2018). Recent advances in convolutional neural networks. Patt. Recogn. 77, 354-377. doi: $10.1016 / j . p a t c o g .2017 .10 .013$

Gulshan, V., Peng, L., Coram, M., Stumpe, M. C., Wu, D., Narayanaswamy, A., et al. (2016). Development and validation of a deep learning algorithm for detection of diabetic retinopathy in retinal fundus photographs. JAMA 316, 2402-2410. doi: 10.1001/jama.2016.17216

He, K., Zhang, X., Ren, S., and Sun, J. (2016). "Deep residual learning for image recognition," in Proceedings of the IEEE Conference on Computer Vision and Pattern Recognition (Beijing), 770-778.

Ho, T. K. (1995). "Random decision forests," in Proceedings of the Third International Conference on Document Analysis and Recognition (Volume 1) Volume 1, ICDAR' 95 (Washington, DC: IEEE Computer Society), 278.

Ioffe, S., and Szegedy, C. (2015). Batch normalization: accelerating deep network training by reducing internal covariate shift. arXiv arXiv:1502.03167.

Isensee, F., Kickingereder, P., Wick, W., Bendszus, M., and Maier-Hein, K. H. (2017). "Brain tumor segmentation and radiomics survival prediction: Contribution to the brats 2017 challenge," in International MICCAI Brainlesion Workshop (Springer), 287-297.
Kamnitsas, K., Bai, W., Ferrante, E., McDonagh, S., Sinclair, M., Pawlowski, N., et al. (2017). "Ensembles of multiple models and architectures for robust brain tumour segmentation," in International MICCAI Brainlesion Workshop (London: Springer), 450-462.

Kingma, D. P. and Ba, J. (2014). Adam: a method for stochastic optimization. arXiv arXiv: 1412.6980

Kingma, D. P., and Ba, J. (2015). "Adam: a method for stochastic optimization," in International Conference on Learning Representations (Amsterdam).

Liu, L., Zhang, H., Wu, J., Yu, Z., Chen, X., Rekik, I., et al. (2018). Overall survival time prediction for high-grade glioma patients based on large-scale brain functional networks. Brain Imaging Behav. 1-19. doi: 10.1007/s11682-018-9949-2

Menze, B. H., Jakab, A., Bauer, S., Kalpathy-Cramer, J., Farahani, K., Kirby, J., et al. (2015). The multimodal brain tumor image segmentation benchmark (brats). IEEE Trans. Med. Imag. 34:1993-2024. doi: 10.1109/TMI.2014.2377694

Milletari, F., Navab, N., and Ahmadi, S.-A. (2016). "V-net: fully convolutional neural networks for volumetric medical image segmentation," in 2016 Fourth International Conference on 3D Vision (3DV), (Munich: IEEE) $565-571$.

Polikar, R. (2006). Ensemble based systems in decision making. IEEE Circ. Syst. Magaz. 6, 21-45. doi: 10.1109/MCAS.2006.1688199

Ronneberger, O., Fischer, P., and Brox, T. (2015). "U-net: convolutional networks for biomedical image segmentation," in International Conference on Medical Image computing and Computer-Assisted Intervention (Freiburg: Springer), 234-241.

Sharma, N., and Aggarwal, L. M. (2010). Automated medical image segmentation techniques. J. Med. Phys. Assoc. Med. Phys. India 35:3-14. doi: 10.4103/0971-6203.58777

Sun, L., Zhang, S., and Luo, L. (2018). "Tumor segmentation and survival prediction in glioma with deep learning," in International MICCAI Brainlesion Workshop (Shenzhen: Springer), 83-93.

Ulyanov, D., Vedaldi, A., and Lempitsky, V. (2016a). Instance normalization: The missing ingredient for fast stylization. arXiv arXiv:1607.08022.

Ulyanov, D., Vedaldi, A., and Lempitsky, V. (2016b). Instance normalization: the missing ingredient for fast stylization. arxiv 2016. arXiv arXiv:1607. 08022.

Van Griethuysen, J. J. M., Fedorov, A., Parmar, C., Hosny, A., Aucoin, N., Narayan, V., et al. (2017). Computational radiomics system to decode the radiographic phenotype. Cancer Res. 77, e104-e107. doi: 10.1158/0008-5472.CAN17-0339

Wang, G., Li, W., Ourselin, S., and Vercauteren, T. (2017). "Automatic brain tumor segmentation using cascaded anisotropic convolutional neural networks," in International MICCAI Brainlesion Workshop (London: Springer), 178-190.

Conflict of Interest Statement: The authors declare that the research was conducted in the absence of any commercial or financial relationships that could be construed as a potential conflict of interest.

Copyright (ㄷ) 2019 Sun, Zhang, Chen and Luo. This is an open-access article distributed under the terms of the Creative Commons Attribution License (CC BY). The use, distribution or reproduction in other forums is permitted, provided the original author(s) and the copyright owner(s) are credited and that the original publication in this journal is cited, in accordance with accepted academic practice. No use, distribution or reproduction is permitted which does not comply with these terms. 